\title{
Russian scintillation PMTs for photon detectors developed and manufactured by MELZ
}

\section{Sergey Belyanchenko ${ }^{1}$}

MELZ FEU Ltd

124460 Russia, Moscow, Zelenograd, pr-d 4922, 4, str. 4.

E-mail:bel-pmt@ya.ru

\section{Valentin Ilievskiy}

MELZ FEU Ltd

124460 Russia Moscow, Zelenograd, pr-d 4922, 4, str. 4.

E-mail:bel-pmt@ya.ru

\section{Ilya Silaev}

MELZ FEU Ltd

124460 Russia Moscow, Zelenograd, pr-d 4922, 4, str. 4.

E-mail:bel-pmt@ya.ru

\section{Vladimir Rykalin}

Institute of High Energy Physics

142281 Russia, Moscow Region, Protvino, pl. Nauki, 1

E-mail: vladimir.rykalin@ihep.ru

\section{Bayarto Lubsandorzhiev}

Institute for Nuclear Research of the Russian Academy of Sciences

117312 Russia Moscow, pr-t 60-letiya Oktyabrya $7 a$.

E-mail:lubsand@rambler.ru

\section{Sultim Lubsandorzhiev}

Institute for Nuclear Research of the Russian Academy of Sciences

117312 Russia Moscow, pr-t 60-letiya Oktyabrya $7 a$.

E-mail:sultim@inbox.ru

Photomultiplier tubes (PMT) are used actively in overwhelming majority of physics experiments. Wide application of photon detectors in high-energy physics, cosmic ray physics, astrophysics as well as security systems and medical diagnostics requires huge variety of PMT types both by dimensions and parameters. "MELZ FEU" Ltd. is a leading Russian designer and manufacturer of various types of PMTs with high sensitivity, low noise level, high-speed impulse counting and excellent energy resolution.

International Conference on New Photo-detectors

PhotoDet 2015

6-9 July 2015

Moscow, Troitsk, Russia

\section{${ }^{1}$ Speaker}

(c) Copyright owned by the author(s) under the terms of the Creative Commons 


\section{Introduction.}

One of the greatest physicists of all times and the founder of the general theory of relativity said: "The most beautiful thing we can experience is the mysterious. It is the source of all true art and science. He to whom the emotion is a stranger, who can no longer pause to wonder and stand wrapped in awe, is as good as dead - his eyes are closed" [1].

Moscow Electro-Lamp Plant (MELZ) is the oldest enterprise of Soviet and Russian electronic industry, the founder of a serial production of incandescent lamps in Russia. MELZ started its historical way in 1906 from a small electro-lamp workshop and in two years became a factory that manufactured 300 thousand bulbs per year. In 1913 the factory merged with another bulb factory called "Light" that worked in Moscow since 1910. The united manufacture was named "Russian electric lamp." In 1921, the Moscow Association of Electro-Lamp Factories was established. It was instigation for restoration of the whole industry after havocs of Russian civil war. Within the association chemical, experimental and industrial laboratories were opened, as well as technical department and design office were established. Thus by 1929, all electro-lamp manufactures were united under one roof. July 27, 1931 MELZ was visited by Bernard Shaw (author of the famous Pygmalion), where the writer talked with the workers and especially with the representatives of literary circles.

During 1930s MELZ mastered production of tantalum. Moreover a new type of vacuum electro-lamps with spiral filament body had been developed and introduced; a number of new light sources and gas discharge devices had been mastered. Specialists of MELZ prepared and implemented into manufacturing process a super hard alloy called "pobedit" and domestic substitute for platinum-platinum bimetal. In 1940 company exported 5 million electric light bulbs to England. In 1942 a dedicated workshop for radio tubes had been set up. It started manufacturing cathode-ray tubes for radio-locators. In 1946, on the basis of cathode ray tubes department a special design bureau (OKB MELZ) was established.

In 1955 a conveyer production line for luminescence lamps had been developed. By 1965, reconstruction of production areas of the plant was finished. Capability of the production facilities increased more than twice. Finally, in 1969, new association was named MELZ. Production range included about 800 units at that time. In 1974, experts of MELZ started manufacturing missile guidance systems. And in 1977 mass production of image intensifier tubes and photo-multiplier tubes was initiated. In 1979 the plant launched pilot production of optical lasers. By 1980, MELZ products had already been exported to more than thirty countries and its association included more than 10 industrial factories.

The new process of thinking of the end of XX century and reconstruction of all the government system made a strong impact upon the activity of the plant. Expansion of market relations began to influence more and more on economic indicators of enterprise and demanded both modification of management structure and changing of ordinary rhythm of work. Destruction of subsidiaries, increasing energy and logistics charges had brought to essential reduction of manufacturing capabilities. Large production premises were not used any longer. Quite a number of light devices were eliminated from the nomenclature of the enterprise. It was forced decision to redirect powerful glass shop from manufacturing glass components for cathode-ray tubes to making glass bottles. During some time this step allowed to improve 
economic situation of the plant and to support manufacturing of traditional directions of MELZ activity including its simultaneous reorganization in research and production complex (RPC). Created in the middle of 90th RPC managed not only to overcome negative influence of economic upheavals but also began a total reconstruction of those production areas which made glory of MELZ. MELZ became a Joint Stock Company in 1996 and was reorganized into Limited Liability Corporation at the beginning of 2008.

Thus, nowadays MELZ is not only a symbiosis of century old experience and up-to-date technology that fully corresponds to leading international standards but also a team of dedicated professionals!

\section{Products}

Our product line is quite vast and able to fulfill variable customer's requirements, but generally, company's policy is focused on manufacturing following types of products: image intensifier tubes (IIT) of 2 and $2+$ generations, developed both for night vision devices of military, special and civilian applications, and for implementation of scientific and research tasks; photomultiplier tubes (PMT) with high sensitivity, low noise level, high-speed impulse counting and excellent energy resolution for medical diagnostics, photon detectors' applications in high-energy physics, cosmic ray physics, astrophysics and also for geophysical and nuclear research, photo- and radio spectroscopy.

\subsection{Image Intensifier Tubes (IITs)}

IITs of $2 \mathrm{~d}$ generation ( $25 \mathrm{~mm}$ photocathode diameter);

IITs of $2+$ generation ( $18 \mathrm{~mm}$ photocathode diameter);

Ultraviolet image intensifiers;

IITs with black\&white screen emission color ( $2 \mathrm{~d}$ and $2+$ generations);

IITs combined with a photosensitive matrix (CCD), which allow to receive and process digital output signal.

\subsection{Night Vision Devices}

Binoculars;

monoculars;

goggles;

scopes.

\subsection{Photomultiplier tubes}

High-temperature PMTs;

Ultraviolet PMTs;

PMTs for photon counting techniques;

Micro-channel plate PMTs;

Compact PMTs with special protection from external magnetic fields. 
Here we will briefly describe some recent developments of PMTs for application in scintillation detectors. Relatively small PMT - FEU-85BH has bialkali photocathode $(25 \mathrm{~mm}$ in diameter) with $>60 \mathrm{~mA} / \mathrm{W}$ spectral sensitivity at $\lambda=420 \mathrm{~nm}$ which allowed to improve considerably the energy resolution of scintillation detectors with this PMT. FEU-85BH rigid pin base compatible with standard E678 -14C socket. Fig. 1 shows picture of FEU-85BH. The PMT has 11 linear focused dynodes. Manufacturing technology of FEU 85BH was designed taking into account operating voltages of PMT in the range of 550-750V.

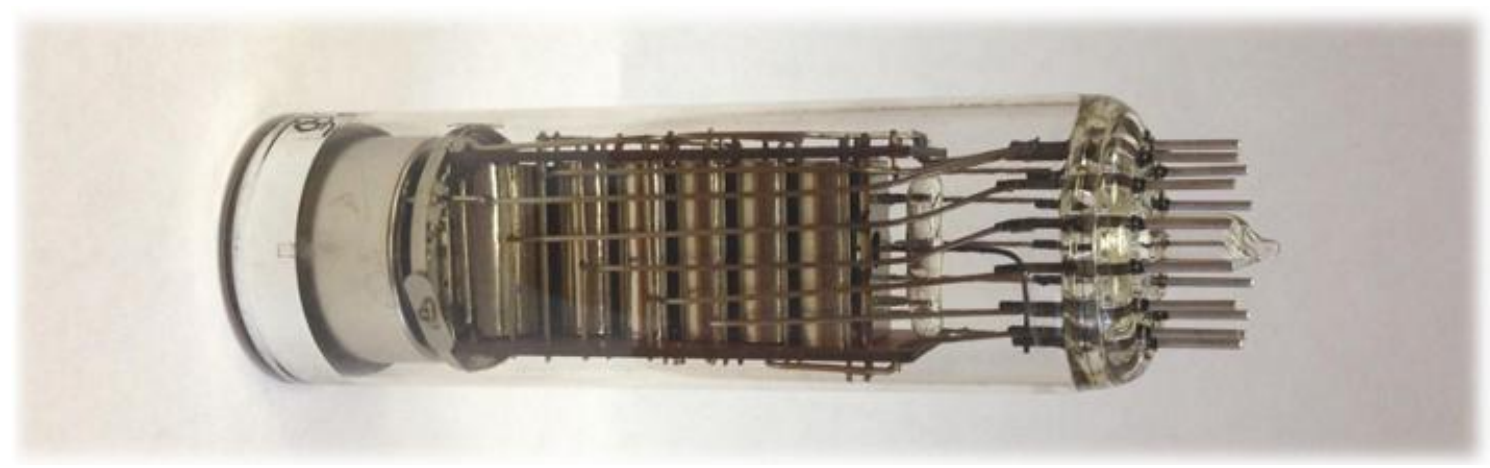

Fig. 1. Picture of FEU-85BH PMT.

As regards to FEU 85BH, the above comparisons, particularly dark characteristics, gain, signal-to-noise ratio, give us an opportunity to claim that performances of FEU 85BH does not differ much from R6094 and is constructively interchangeable for it.

One of the state-of-the-art developments of MELZ is a PMT with the 3 inch hemispherical bialkali photocathode, Fig. 2. The PMT is presently under development for use in low energy neutrino experiments like the ORCA project in the Mediterranean [2]. Low energy neutrino experiments comprise Cherenkov detectors and liquid scintillator detectors as well. For the ORCA project 3 inch PMTs will instrument 17 inches in diameter spherical deep underwater optical module. In case of liquid scintillators PMTs will be immersed directly into liquid scintillator to provide better reconstruction of neutrino events, see [3] for references.

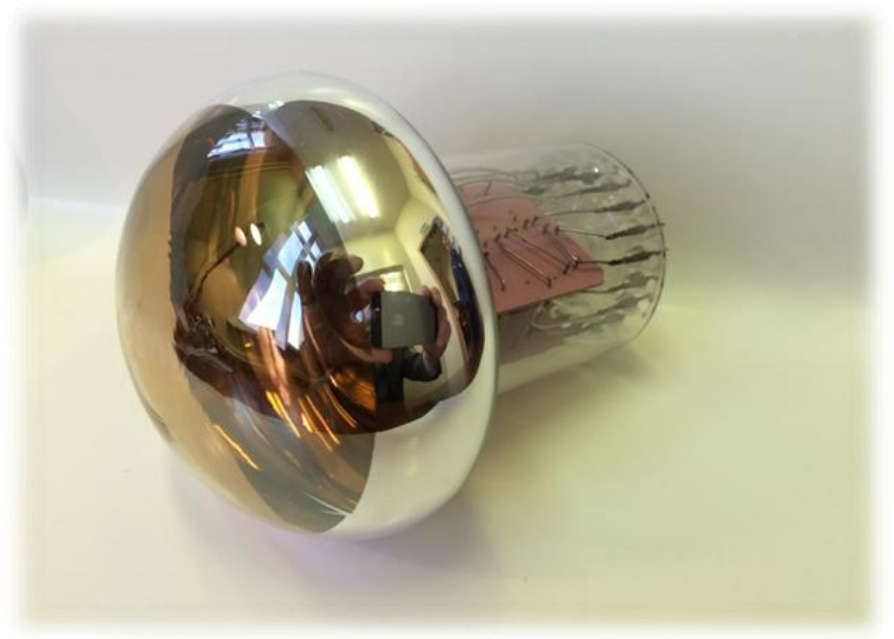

Fig. 2. 3-inch PMT with mushroom shape glass envelope developed for low energy neutrino experiments. 
In this PMT we used an electro-optical system of linear type with 10 trough-shaped dynodes with deposited emitters fixed on ceramic supporting plates. The mesh anode located between two last dynodes is used to increase the linearity of output signals. Spectral response range of bialkali photocathode is $320-650 \mathrm{~nm}$. Spectral sensitivity at $405 \mathrm{~nm}$ wavelength is not less than $130 \mathrm{~mA} / \mathrm{W}$. The PMT's gain of $5 \times 10^{6}$ is reached at operating voltage less than $1600 \mathrm{~V}$. Transit time spread of single photoelectron pulses (TTS) does not exceed 3 ns (FWHM). In Fig. 3 the TTS spectrum of 3" PMT measured at 0.25 p.e. threshold and $5 \times 10^{6}$ gain is shown.

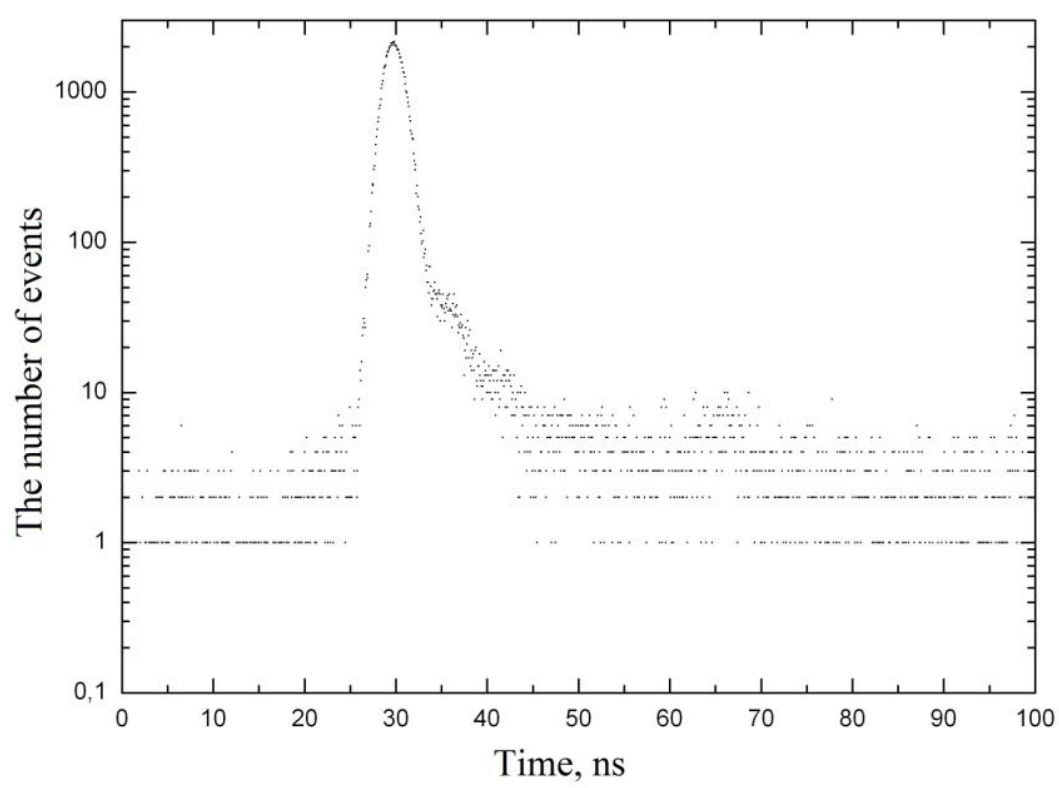

Fig. 3. Spectrum of transit time spread of single photoelectron pulses (TTS) measured for 3" PMT with hemispherical photocathode.

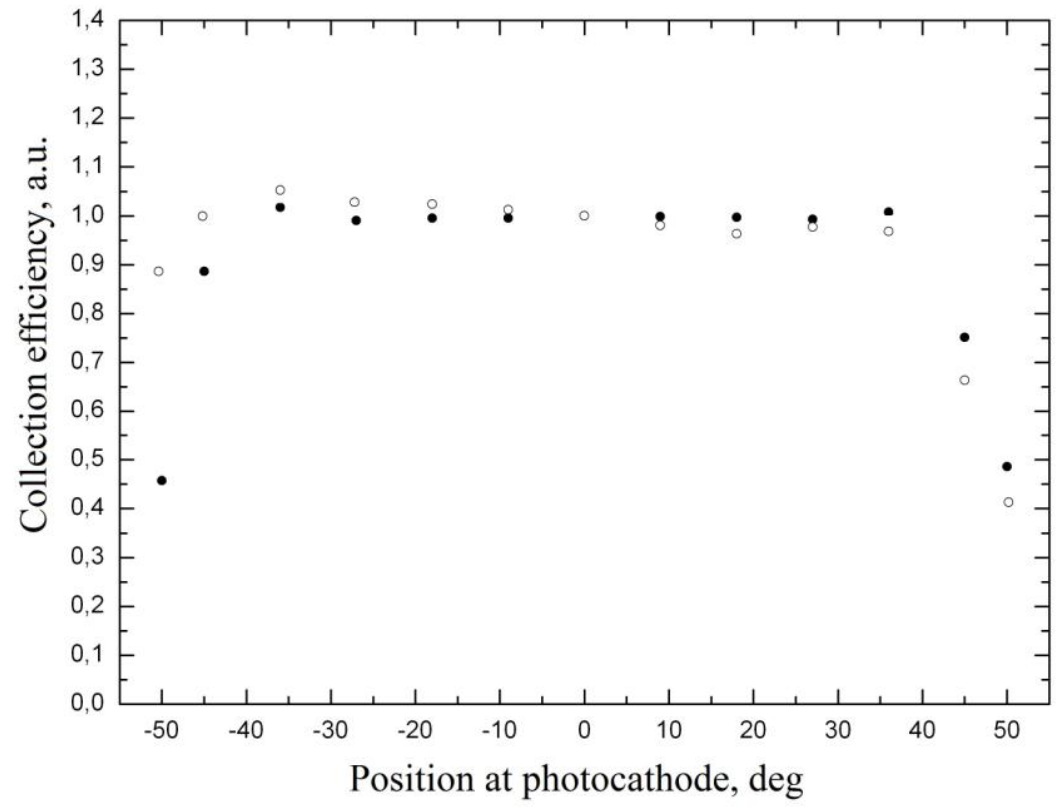

Fig. 4. Dependence of collection efficiency on the position of illumination at the PMT's photocathode measured for two perpendicular directions. 
The PMT is constructed in a mushroom shaped glass envelope having $76 \mathrm{~mm}$ in diameter and $43.2 \mathrm{~mm}$ curvature radius. Overall length without pins is $105 \mathrm{~mm}$, external diameter of the neck $-52 \mathrm{~mm}$. This optimal radius of photocathode curvature $(43.2 \mathrm{~mm})$ was calculated by means of computer modeling in order to increase photoelectron collection efficiency at the minimum of TTS. Dependence of collection efficiency on the position of illumination at the PMT's photocathode measured for two perpendicular directions (one of them was oriented along the first dynode surface) is demonstrated in Fig. 4.

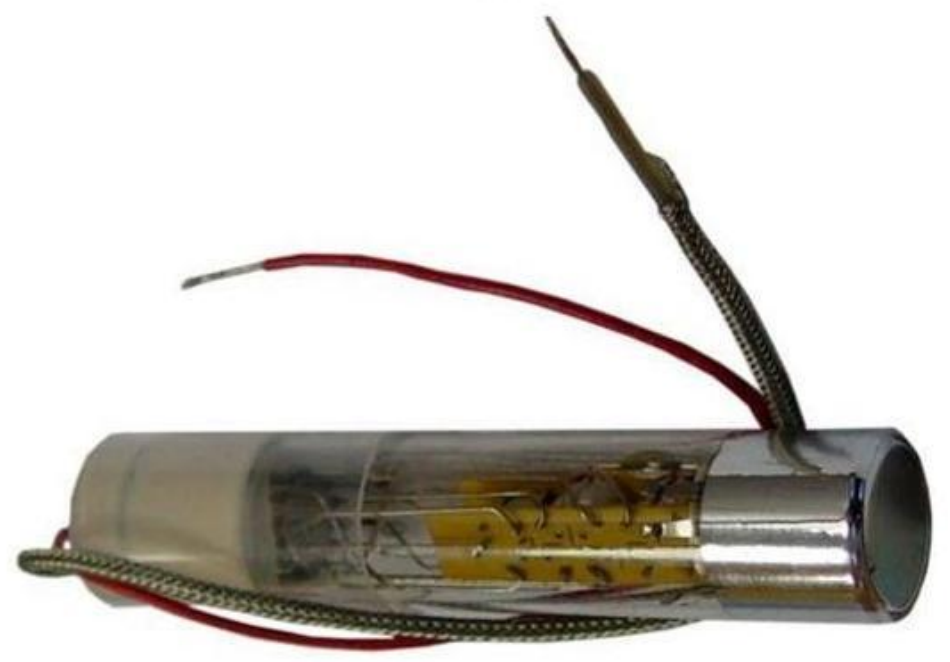

Fig. 5. Picture of FEU-86 PMT developed for photon counting application.

FEU-86U, Fig. 5, with potted voltage divider is also of interest to consumers. It has $16 \mathrm{~mm}$ photocathode diameter with maximum $22 \mathrm{~mm}$ of overall diameter, weighting not more than 50 g. Luminous noise equivalent of the dark current of the anode is not more than $5 \times 10^{-13} \mathrm{~mm} / \mathrm{Hz}^{1 / 2}$, and the dark current counting rate at threshold of 0.25 p.e. is less than $300 \mathrm{~s}^{-1}$. All this parameters in the aggregate allow us to consider FEU-86U as one of the best photomultiplier manufactured in Russia for photon counting application. Presently the PMT is widely used in portable monitoring equipment for air environment in enclosed spaces.

Two types of PMTs for operation at high temperatures have been developed by MELZ FEU, namely FEU-74A and FEU-158. The former operates at temperatures till $150^{\circ} \mathrm{C}$ and the latter can withstand temperatures as high as $200^{\circ} \mathrm{C}$. Both types of PMTs are widely used in geophysics studies. Presently development of other types of high temperature PMTs with improved parameters is actively being done. 
Usually the sizes of plastic scintillators of gamma radiation monitors for portal detectors are around 1 meter long and $50 \mathrm{~mm}$ thick. Scintillators are viewed by 2-inch PMT. Photocathode area of that PMT is less than $20 \mathrm{~cm}^{2}$ thus is light collection efficiency is conspicuously small.

In turn it results in low efficiency for gamma quanta detection in the energy range lower than $100 \mathrm{\kappa eV}$. To solve the problem we came up to an idea of development of PMT with an extended cylindrical photocathode that allows to improve light collection significantly. In the upper part of Fig. 6 the picture of the PMT is shown. In the bottom part of Fig. 6 one can see the PMT fixed in plastic scintillator slab. With a length of photocathode of $200 \mathrm{~mm}$ and its diameter of $40 \mathrm{~mm}$ the active sensitive area of the PMT exceeds the sensitive area of conventional 2-inch PMT by a factor of more than 10. The maximum distance passed by photons decreases twice in comparison with conventional PMT and besides, the photons hitting PMT and not absorbed in the first encountering with the PMT photocathode can hit its photocathode on the opposite side, thus increasing effective sensitivity of the PMT considerably.
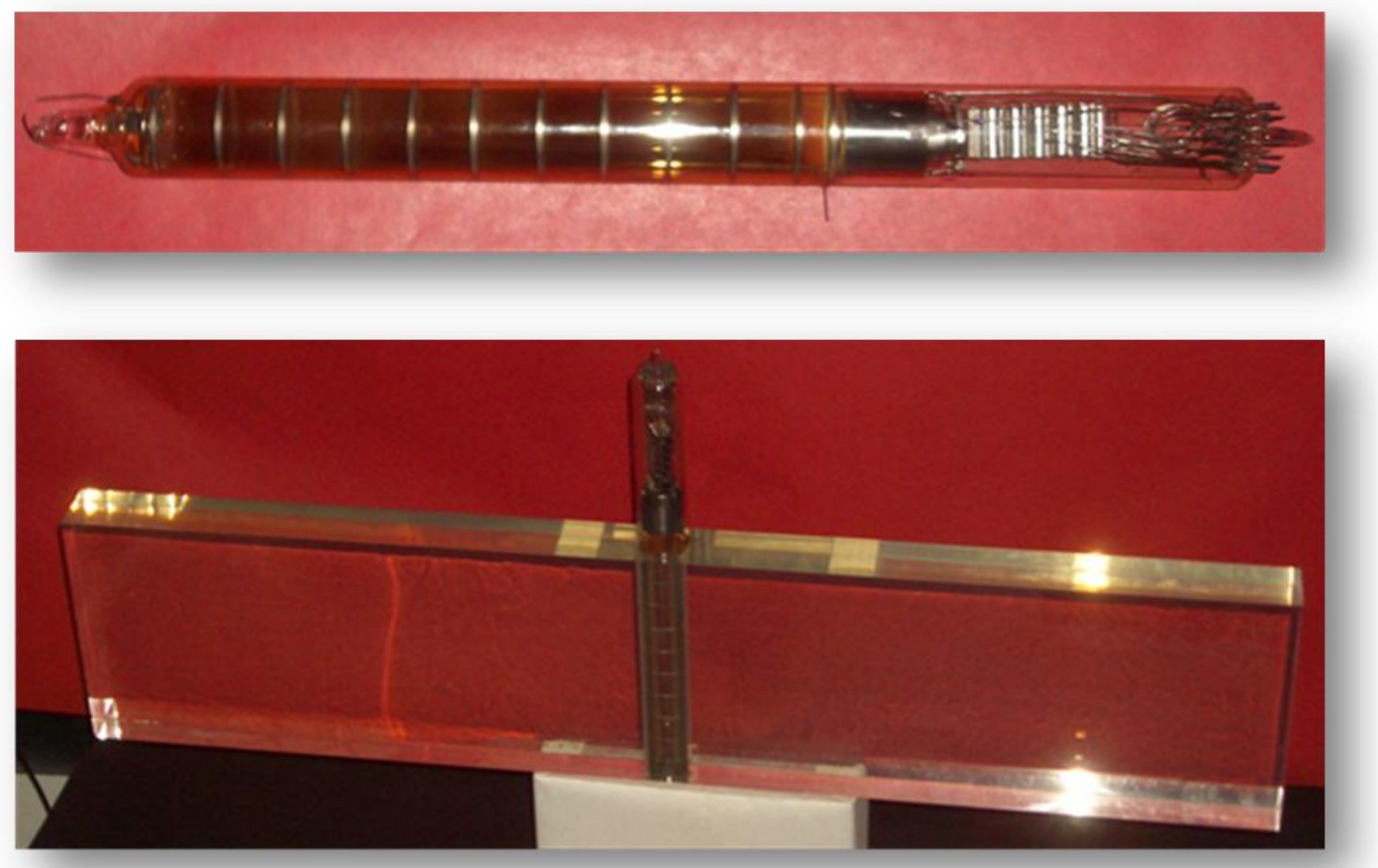

Fig. 6. Up - a PMT with cylindrical photocathode; Bottom - the PMT in plastic scintillator.

We think that nowadays interest to those photomultipliers appears again. It is connected with creation of big neutrino liquid scintillating detectors with axial symmetry. Length of these detectors is a few meter, diameter - more than one meter. The arrangement with a chain of that PMT along an axis of symmetry of the detector is optimum by efficiency and uniformity of a light collection. Up to now we developed a new design of venetian blind dynodes, produced some models and the first prototype of the new PMT with photocathode sizes of $\varnothing 60 \times 200 \mathrm{~mm}^{2}$ 
(take a look at bottom pictures). The reached level of blue sensitivity is $30-40 \mathrm{~mA} / \mathrm{W}$, multiplication gain $-10^{6}$ as with 13 venetian blind dynodes.

Currently, we are working on developing and manufacturing of photomultipliers based on FEU 85 and FEU 115M with flat-concave input window. They will be produced with bialkali photocathode with different number of gain stages and corresponding connection leads which fully correspond to both Russian and international standards (like FEU 85BH). That will allow us to realize import substitution program to the full extent.

\section{Conclusion.}

Photomultipliers developed by MELZ FEU Company are used presently in high energy physics, astrophysics, medical studies, as well as in many other areas of science and industry and they are able to meet wide range of requirements put by present day and future physics experiments.

\section{Acknowledgements.}

The authors are indebted very much to Dr. V.Ch. Lubsandorzhieva for careful reading of the manuscript, invaluable discussions and many useful corrections.

\section{References}

[1] A. Einstein. Living Phylosophies. New York: Simon and Schuster, 1931.

[2] P. Kooijman et al. (KM3NeT Collaboration). Multi-PMT Optical Module // Nuclear Instruments and Methods A. 2006. V.567. P.508.

[3] T. Adam et al. (JUNO Collaboration). JUNO conceptual design report // arxiv:1508.07166. 